\title{
Phytochemical Screening and In-Vitro Antioxidant Activity of Peristrophe paniculata
}

\section{Srikanth M*, Devi B, Kotirataiah K, Ramanjaneyulu M, Sulthana PN and Suma RR}

Department of Pharmacognosy, Medarametla Anjamma Mastanrao College of Pharmacy, Narasaraopet, Andhra Pradesh, India

${ }^{*}$ Corresponding author: Srikanth M, Department of Pharmacognosy, Medarametla Anjamma Mastanrao College of Pharmacy, Narasaraopet, Andhra Pradesh, India, Tel: 9492723149; E-mail: drsrikathphd2014@gmail.com

Received date: March 27, 2018; Accepted date: April 06, 2018; Published date: April 12, 2018

Copyright: (C) 2018 Srikanth M, et al. This is an open-access article distributed under the terms of the Creative Commons Attribution License, which permits unrestricted use, distribution, and reproduction in any medium, provided the original author and source are credited.

Citation: Srikanth M, Devi B, Kotirataiah K, Ramanjaneyulu M, Sulthana PN, et al. Phytochemical Screening and In-Vitro Antioxidant Activity of Peristrophe paniculata. Herb Med. 2018, Vol.4 No.1:1.

\section{Abstract}

Peristrophe paniculata is one of the traditional medicinal plant have been using in treatment of different diseases. The present study was carried out to provide scientific evidence about its medicinal use and phytochemical variation in different parts (stem, leaves and root) of it. Phytochemical studies were carried out for hexane, ethyl acetate and hydro alcoholic extracts using standard test procedures and antioxidant activity was carried on different free radicals i.e., superoxide, hydroxyl and 1, 1diphenyl-2-picrylhydrazyl (DPPH). Hydroalcoholic, ethyl acetate and hexane extracts of $P$. paniculata were found to possess concentration dependent free radical scavenging activity on superoxide, hydroxyl and DPPH free radicals. Qualitative phytochemical screening of $P$. paniculata extracts revealed the presence of diverse in phytochemical constituents like steroids, terpenoids, flavonoids, alkaloids, glycosides, phenols, tannins and carbohydrates. The extracts of different parts gave negative and positive results for the amino acids, oils and saponins. The selected plant extracts showed concentration dependent percentage of inhibition on tested free radicals along with the standard drug ascorbic acid. The hexane extract of all parts of $P$. paniculata showed lower activity compared to ethyl acetate and hydroalcoholic extracts. Hydroalcoholic extract showed better activity. The variation in the activity and phytochemical constituents in them maybe due to the compounds present in them either as individually or in mixtures. The further research is need to evaluate more pharmacological activities and in isolation of the bioactive compounds from $P$. paniculata.

Keywords: $P$. paniculata; $\quad$ Stem; Leaves; Roots; Phytochemical analysis; Antioxidant activity

\section{Introduction}

Medicinal plants play a central role in traditional medicines and are precursors for the modern pharmaceuticals/Allopathic medicines. Most of the natural products found in medicinal plants are the compounds biosynthetically derived from primary metabolites such as amino acids, carbohydrates and fatty acids and are generally categorized as secondary metabolites [1,2]. The high technology tests such as computer tomography scans, magnetic resonance imaging, endoscopies, radiotherapies and biopsies are expensive and often treatments frequently have side effects such as allergies, vomiting, headaches etc. whereas traditional remedies usually have none $[3,4]$. So, the traditional medicine is largely gaining popularity over allopathic medicine because of rising costs of medical care, free from side effects in several cases, easy availability of drugs from natural sources and etc. Different drugs from different parts of different plant sources shows various therapeutic activities and different biologically and pharmacologically active compounds were identified $[5,6]$.

From earliest times and even today almost all drugs are intimately linked with plants and these plant products have extensive use in the ethno-medicine and traditional system of medicine. The untapped wealth of plant kingdom increased interest on medicinal plants enormously over last three decades. Thereby become target for the search of new drugs and lead molecules for treatment of different diseases [7-9]. In recent years, a comprehensive search on medicinal plants, pharmaceutical drugs recently published in clinical reports suggest the effectiveness of herbal remedies, their availability, low cost. Thus, the present study was carried out on evaluation of In-vitro antioxidant potential of different extracts of Peristrophe paniculata.

Peristrophe paniculata (Peristrophe bicalyculata) belongs to family Acanthaceae, is an erect, perennial plant with stems that can become more or less woody; it can grow up to $150 \mathrm{~cm}$ tall. Widespread through subtropical and tropical Africa, E. Asia-China, India, Nepal, Myanmar, through tropical Asia to Australia. Forest undergrowth, hedges, and wasteland. Weedy areas, roadsides at elevations from 600-2,200 meters [10]. The 
plant $P$. paniculata can be used as a green manure and has medicinal potential. Leaf juice have been used for healing fractured bones and plant material maceration used as antidote to snake poison and the plant material was also used for treatment of hypertension, cardiovascular diseases [9-13]. There is very few phytochemical and biological works were reported on $P$. paniculata [14-17].

\section{Materials and Methods}

\section{Chemicals and drugs}

The chemicals and solvents used in the current study were analytical grade from S.D. Fine Chemicals Pvt. Ltd., Mumbai. 1,1-diphenyl-2-picrylhydrazyl (DPPH) from Sigma Chemical Company, U.S.A., Riboflavin from Loba Chemic., Mumbai. Deoxyribose from Sisco Research Laboratories Pvt Ltd., Mumbai, Nitroblue tetrozolium from Sisco Research Laboratories Pvt Ltd., Mumbai.

\section{Collection of plant material and preparation of extracts}

The plant material was collected at Palnadu region, Andhra Pradesh, India, during the month November, 2017. The authentication of the plant was done by Rtd. Dr. Prayaga Murthy. Pragada, Department of Botany, Government Degree College, Yeleswaram, E. Godavari, Andhra Pradesh. Different parts of the plant were separated and shade dried. Then, plant materials were powdered and separately extracted using maceration process with hexane, ethyl acetate, and hydroalcoholic (ethanol $(70 \% \mathrm{v} / \mathrm{v})$ ) were concentrated to dryness under vacuum using rotavapour.

\section{Phytochemical analysis}

Phytochemical studies were carried out for hexane, ethyl acetate and hydro alcoholic extracts of $P$. paniculata stem, leaves and roots separately to detect the presence of different phytochemical constituents like steroids, terpenoides, tannins, flavanoids, saponins, glycosides, amino acids etc. by using standard procedures [18-20].

\section{In vitro antioxidant activity}

For the assessment of antioxidant activity, the extract of B. roxburghii was dissolved in Dimethyl sulphoxide (DMSO). The results were showed in mean \pm SEM. The Percentage Inhibition and $50 \%$ Inhibition Concentration's (IC50) were calculated $[18,21]$. The each values were studied against blank in every assay of scavenging activity.

\section{Superoxide radical scavenging activity}

Superoxide scavenging activity of the selected plant extract was evaluated as per method [22]. It is by absorption of light at $560 \mathrm{~nm}$ induction of superoxide free radical generation by riboflavin and corresponding reduction by nitroblue tetrazolium.

\section{Hydroxyl radical scavenging activity}

The hydroxyl radical scavenging activity is measured as per established method. It was studied by the competition between deoxyribose and the extract's antioxidant molecules for hydroxyl radicals generated from the $\mathrm{Fe}^{+2} / \mathrm{EDTA} / \mathrm{H} 2 \mathrm{O} 2$ system [23].

\section{2,2-Diphenyl-1-picrylhydrazyl (DPPH) radical scavenging activity}

The DPPH radical scavenging activity was measured as per methods [24,25]. This method is based on measure of color absorbance of alcoholic DPPH solution (Blue color) after addition of antioxidant solution (Extract/Compound). If antioxidants present in the test compound blue color yellow color due to diphenyl-picrylhydrazine.

\section{Calculation of percentage inhibition}

The percentage inhibition of superoxide production by the extract was calculated using the formula: Inhibitory ratio $=(\mathrm{A} 0$ A1)/A0+100

Where, A0 is the absorbance of control; A1 is the absorbance with addition of plant extract/ ascorbic acid.

\section{Calculation of $50 \%$ inhibition concentration}

The optical density obtained with each concentration of the extract/ascorbic acid was plotted taking concentration on Xaxis and percentage inhibition on Y-axis. The graph was extrapolated to find the $50 \%$ inhibition concentration of extract/ ascorbic acid.

\section{Results}

\section{Qualitative phytochemical screening of Peristrophe paniculata stem extracts}

Qualitative phytochemical screening of $P$. paniculata stem extracts revealed the presence of different phytochemical constituents The divergence was found in the presence and absence phyto-compounds (Table 1). All the extracts revealed the presence of only carbohydrates and absence for quinones. The hexane extract gave positive results for the steroids, terpenoids, carbohydrates and oils but gave negative results for glycosides, saponins, flavanoids, alkaloids, tannins, phenols, amino acids and quinones. The ethyl acetate extract revealed the presence of terpenoids, glycosides, carbohydrates, flavanoids, alkaloids, tannins and amino acids but gave negative results for steroids, saponins, phenols, oils and quinones. The hydroalcoholic extract revealed the presence of steroids, glycosides, saponins, carbohydrates, 
alkaloids, tannins, phenols and amino acids but gave negative results for terpenoids, flavanoids, oils and quinones.

Table 1 Nature of phytoconstituents in stem extract of Peristrophe paniculata.,$+++=$ Present, $-=$ Absent.

\begin{tabular}{|c|c|c|c|}
\hline \multirow[t]{2}{*}{ Phytochemical constituents } & \multicolumn{3}{|c|}{ Peristrophe paniculata stem } \\
\hline & Hexane extract & Ethyl acetate extract & Hydro alcoholic extract \\
\hline Sterols & + & - & + \\
\hline Terpenoids & + & + & - \\
\hline Glycosides & - & + & ++ \\
\hline Saponins & - & - & + \\
\hline Flavonoids & - & ++ & - \\
\hline Alkaloids & - & ++ & ++ \\
\hline Tannins & - & + & + \\
\hline Carbohydrates & + & + & + \\
\hline Phenols & - & - & ++ \\
\hline Oils & + & - & - \\
\hline Amino acids & - & + & + \\
\hline Quinones & - & - & - \\
\hline
\end{tabular}

\section{Qualitative phytochemical screening Peristrophe paniculata leaves extracts}

Qualitative phytochemical screening of $P$. paniculata leaves extracts revealed the presence of different phytochemical constituents. The difference was found in the presence and absence phyto-compounds (Table 2). All the extracts revealed the presence of only sterols, carbohydrates. The hexane extract gave positive results for the steroids, terpenoids, glycosides, flavanoids, carbohydrates and oils but gave negative results for saponins, alkaloids, tannins, phenols, amino acids and quinones. The ethyl acetate extract revealed the presence of steroids, terpenoids, glycosides, carbohydrates, flavanoids, phenols, tannins, amino acids and quinones but gave negative results for alkaloids, saponins and oils. The hydroalcoholic extract revealed the presence of steroids, glycosides, saponins, carbohydrates, alkaloids, tannins and phenols but gave negative results for terpenoids, flavanoids, amino acids, oils and quinones.

Table 2 Nature of phytoconstituents in leaves' extract of Peristrophe paniculata. +, ++=Present, - =Absent (+=Less Intense; ++=More Intense).

\begin{tabular}{|l|l|l|l|}
\hline \multirow{2}{*}{$\begin{array}{l}\text { Phytochemical } \\
\text { constituents }\end{array}$} & \multicolumn{3}{|c|}{ Peristrophe paniculata leaves } \\
\cline { 2 - 4 } & $\begin{array}{l}\text { Hexa } \\
\text { ne } \\
\text { extra } \\
\text { ct }\end{array}$ & $\begin{array}{l}\text { Ethyl acetate } \\
\text { extract }\end{array}$ & $\begin{array}{l}\text { Hydro alcoholic } \\
\text { extract }\end{array}$ \\
\hline Sterols & + & + & + \\
\hline Terpenoids & + & + & - \\
\hline Glycosides & + & + & + \\
\hline
\end{tabular}

\begin{tabular}{|l|c|c|c|}
\hline Saponins & - & - & + \\
\hline Flavonoids & + & + & - \\
\hline Alkaloids & - & - & + \\
\hline Tannins & - & + & + \\
\hline Carbohydrates & + & + & + \\
\hline Phenols & - & + & + \\
\hline Oils & + & - & - \\
\hline Amino acids & - & + & - \\
\hline Quinones & - & + & - \\
\hline
\end{tabular}

\section{Qualitative phytochemical screening of Peristrophe paniculata root extracts}

Qualitative phytochemical screening of $P$. paniculata root extracts revealed the presence of different phytochemical constituents. The differentiation was found in the presence and absence of phyto-compounds (Table 3 ). All the extracts revealed the presence of terpenoids, alkaloids, phenols and absence for oils, amino acids, carbohydrates, quinones. The hexane extract gave positive results for the steroids, terpenoids, alkaloids, phenols but gave negative results for saponins, tannins, glycosides, flavanoids, carbohydrates, oils, amino acids, and quinones. The ethyl acetate extract revealed the presence of terpenoids, glycosides, flavanoids, alkaloids, phenols, but gave negative results for steroids, carbohydrates, tannins, saponins, oils, amino acids and quinones. The hydroalcoholic extract revealed the presence of steroids, terpenoids, glycosides, carbohydrates, alkaloids, tannins and 
phenols but gave negative results for saponins, flavanoids, amino acids, oils and quinones.

Table 3 Nature of phytoconstituents in root extract of Peristrophe paniculata. +, ++=Present, - =Absent (+=Less Intense; ++=More Intense).

\begin{tabular}{|l|l|l|l|}
\hline \multirow{2}{*}{$\begin{array}{l}\text { Phytochemical } \\
\text { constituents }\end{array}$} & \multicolumn{3}{|c|}{ Peristrophe paniculata leaves } \\
\cline { 2 - 4 } & $\begin{array}{l}\text { Hexane } \\
\text { extract }\end{array}$ & $\begin{array}{l}\text { Ethyl } \\
\text { acetate } \\
\text { extract }\end{array}$ & $\begin{array}{l}\text { Hydro } \\
\text { alcoholic } \\
\text { extract }\end{array}$ \\
\hline Sterols & + & - & + \\
\hline Terpenoids & + & + & + \\
\hline Glycosides & - & + & + \\
\hline Saponins & - & - & - \\
\hline Flavonoids & - & + & - \\
\hline Alkaloids & + & + & + \\
\hline Tannins & - & - & + \\
\hline Carbohydrates & - & - & - \\
\hline Phenols & + & + & + \\
\hline Oils & - & - & - \\
\hline Amino acids & - & - & - \\
\hline Quinones & - & - & - \\
\hline
\end{tabular}

\section{Antioxidant activity}

In the present study, hydroalcoholic, ethyl acetate and hexane extracts of $P$. paniculata were found to possess concentration dependent free radical scavenging activity on superoxide, hydroxyl and DPPH free radicals.

\section{Antioxidant activity of Peristrophe paniculata stem extracts}

The stem extracts of $P$. paniculata were showed concentration dependent percentage inhibition on tested free radicals (superoxide, hydroxyl and DPPH). The IC50 values on superoxide radical of hydroalcoholic extract of $P$. paniculata stem and ascorbic acid were found to be $257 \mu \mathrm{g}$ and $140 \mu \mathrm{g}$ respectively (Figure 1 ). The IC50 values on hydroxyl radical of hydroalcoholic and ascorbic acid were found to be $246 \mu \mathrm{g}, 162$ $\mu \mathrm{g}$ respectively. The IC50 values for $\mathrm{DPPH}$ radical of hydroalcoholic, ethyl acetate and hexane extracts of $P$. paniculata were found to be $218 \mu \mathrm{g}, 266 \mu \mathrm{g}$ and for ascorbic acid was $120 \mu \mathrm{g}$. The $P$. paniculata stem extracts showed better scavenging activity on DPPH free radical than superoxide and hydroxyl free radicals. Among all $P$. paniculata stem extracts, the hydroalcoholic extract showed better activity. Hexane and ethyl acetate extracts at low concentration showed minimum inhibition on tested free radicals (Table 4 ).

\section{Antioxidant activity of Peristrophe paniculata leaf extracts}

The $P$. paniculata leaves extracts were produced concentration dependent percentage inhibition on tested free radicals, among the three extracts, hydralcoholic extract showed better activity than remaining extracts. The hydroalcoholic, ethyl acetate and hexane extracts of $P$. paniculata leaves showed modest inhibition of free radicals at $340 \mu \mathrm{g}$ and hydroalcoholic extract showed the better activity at $340 \mu$ i.e., $38.4 \pm 0.68$. The extracts do not showed $50 \%$ inhibition on free radicals at tested concentrations, may they show $50 \%$ inhibition at higher concentration.

The IC50 values for hydroalcoholic extract of $P$. paniculata leaves and ascorbic acid on hydroxyl radical of were found to be $291 \mu \mathrm{g}$ and $162 \mu \mathrm{g}$, hexane extract showed less and ethyl acetate extract showed moderate activity. The IC50 values for of hydroalcoholic and ethyl acetate extracts of $P$. paniculata leaves on DPPH radical were found to be $222 \mu \mathrm{g}, 264 \mu \mathrm{g}$ respectively (Figure 2 ). The IC50 value of ascorbic acid was found to be $120 \mu \mathrm{g}$. Among all extracts hydroalcoholic extract at a concentration of $320 \mu \mathrm{g}$ showed the better scavenging activity on DPPH free radical i.e., $58.2 \pm 0.69$ (Table 5).

Table 4 Concentration dependent percentage inhibition of different extracts of $P$. paniculata stem on superoxide, hydroxyl and DPPH free radicals.

\begin{tabular}{|c|c|c|c|c|c|c|c|c|c|c|c|c|c|c|c|}
\hline \multirow{2}{*}{$\begin{array}{l}\text { Name of the } \\
\text { extracts }\end{array}$} & \multicolumn{5}{|c|}{$\%$ of inhibition on superoxide radical } & \multicolumn{5}{|c|}{$\%$ of inhibition on hydroxyl radical } & \multicolumn{5}{|c|}{$\%$ of inhibition on DPPH radical } \\
\hline & $\begin{array}{l}20 \\
\mu \mathrm{g}\end{array}$ & $\begin{array}{l}40 \\
\mu g\end{array}$ & $\begin{array}{l}80 \\
\mu g\end{array}$ & $\begin{array}{l}160 \\
\mu g\end{array}$ & $\begin{array}{l}32 \\
\mu \mathrm{g}\end{array}$ & $\begin{array}{l}20 \\
\mu \mathrm{g}\end{array}$ & $\begin{array}{l}40 \\
\mu \mathrm{g}\end{array}$ & $\begin{array}{l}80 \\
\mu g\end{array}$ & $160 \mu g$ & $\begin{array}{l}32 \\
\mu \mathrm{g}\end{array}$ & $\begin{array}{l}20 \\
\mu g\end{array}$ & $\begin{array}{l}40 \\
\mu g\end{array}$ & $\begin{array}{l}80 \\
\mu g\end{array}$ & $160 \mu \mathrm{g}$ & $32 \mu \mathrm{g}$ \\
\hline Hexane extract & $\begin{array}{l}0.0 \\
0\end{array}$ & $\begin{array}{l}6.5 \\
\pm \\
0.0 \\
2\end{array}$ & $\begin{array}{l}11 \pm \\
0.3\end{array}$ & $\begin{array}{l}22.4 \pm \\
0.17\end{array}$ & $\begin{array}{l}36 . \\
2 \pm \\
0.3\end{array}$ & $\begin{array}{l}2.4 \\
6 \pm \\
0.3^{ \pm}\end{array}$ & $\begin{array}{l}5.6 \pm \\
0.18\end{array}$ & $\begin{array}{l}12 . \\
3 \pm \\
0.4\end{array}$ & $\begin{array}{l}23.2 \pm \\
0.82\end{array}$ & $\begin{array}{l}42.3 \\
\pm \\
0.39\end{array}$ & $\begin{array}{l}3.6 \pm \\
0.3\end{array}$ & $\begin{array}{l}8.2 \pm \\
1.2\end{array}$ & $\begin{array}{l}15.3 \\
\pm 0.4\end{array}$ & $\begin{array}{l}28.6 \pm \\
0.62\end{array}$ & $\begin{array}{l}45.6 \\
\pm 0.2\end{array}$ \\
\hline $\begin{array}{l}\text { Ethyl } \\
\text { Acetate extract }\end{array}$ & $\begin{array}{l}3.4 \\
\pm \\
0.3 \\
3\end{array}$ & $\begin{array}{l}8.4 \\
\pm \\
0.0 \\
6\end{array}$ & $\begin{array}{l}15.2 \\
7 \\
0.13\end{array}$ & $\begin{array}{l}28.77 \\
\pm 0.68\end{array}$ & $\begin{array}{l}48 \\
4 \pm \\
0.3\end{array}$ & $\begin{array}{l}0.0 \\
0\end{array}$ & $\begin{array}{l}3.5 \pm \\
0.6\end{array}$ & $\begin{array}{l}6.2 \\
\pm \\
0.4 \\
3\end{array}$ & $\begin{array}{l}12.9 \pm \\
0.16\end{array}$ & $\begin{array}{l}28.9 \\
\pm \\
0.63\end{array}$ & $\begin{array}{l}6.2 \pm \\
0.53\end{array}$ & $\begin{array}{l}11.2 \\
\pm \\
0.35\end{array}$ & $\begin{array}{l}20.9 \\
\pm \\
0.43\end{array}$ & $\begin{array}{l}39.2 \pm \\
0.6\end{array}$ & $\begin{array}{l}56.2 \\
\pm \\
0.93\end{array}$ \\
\hline
\end{tabular}




\begin{tabular}{|c|c|c|c|c|c|c|c|c|c|c|c|c|c|c|c|}
\hline $\begin{array}{l}\text { Hydro- } \\
\text { alcoholicextract }\end{array}$ & $\begin{array}{l}8.5 \\
\pm \\
0.3\end{array}$ & $\begin{array}{l}18 . \\
5 \pm \\
1.0\end{array}$ & $\begin{array}{l}21.4 \\
\pm \\
0.28\end{array}$ & $\begin{array}{l}35.6 \pm \\
0.34\end{array}$ & $\begin{array}{l}59 . \\
2 \pm \\
0.6\end{array}$ & $\begin{array}{l}4.8 \\
\pm \\
0.2 \\
9\end{array}$ & $\begin{array}{l}9.5 \pm \\
0.93\end{array}$ & $\begin{array}{l}19 . \\
3 \pm \\
0.2 \\
8\end{array}$ & $\begin{array}{l}40.3 \pm \\
0.43\end{array}$ & $\begin{array}{l}58.2 \\
3 \pm \\
0.82\end{array}$ & $\begin{array}{l}6.3 \pm \\
0.5\end{array}$ & $\begin{array}{l}9.5 \pm \\
0.93\end{array}$ & $\begin{array}{l}19.3 \\
\pm 0.8\end{array}$ & $\begin{array}{l}43.2 \pm \\
0.8\end{array}$ & $\begin{array}{l}60.48 \\
\pm \\
0.93\end{array}$ \\
\hline Ascorbic acid & $\begin{array}{l}5.8 \\
\pm \\
0.3 \\
9\end{array}$ & $\begin{array}{l}11 . \\
6 \pm \\
0.8 \\
9\end{array}$ & $\begin{array}{l}25.7 \\
\pm 1.2\end{array}$ & $\begin{array}{l}58.64 \\
\pm 0.18\end{array}$ & $\begin{array}{l}75 . \\
36 \\
\pm \\
0.7\end{array}$ & $\begin{array}{l}6.3 \\
9 \pm \\
0.8 \\
4\end{array}$ & $\begin{array}{l}11.9 \\
3 \pm \\
0.45\end{array}$ & $\begin{array}{l}23 . \\
6 \pm \\
0.7 \\
1\end{array}$ & $\begin{array}{l}47.89 \pm \\
0.53\end{array}$ & $\begin{array}{l}73.3 \\
4 \\
0.62\end{array}$ & $\begin{array}{l}10.2 \\
6 \pm \\
0.28\end{array}$ & $\begin{array}{l}20.4 \\
7 \pm \\
0.75\end{array}$ & $\begin{array}{l}36.9 \\
\pm \\
0.55\end{array}$ & $\begin{array}{l}62.31 \pm \\
1.26\end{array}$ & $\begin{array}{l}78.08 \\
\pm \\
0.04\end{array}$ \\
\hline
\end{tabular}

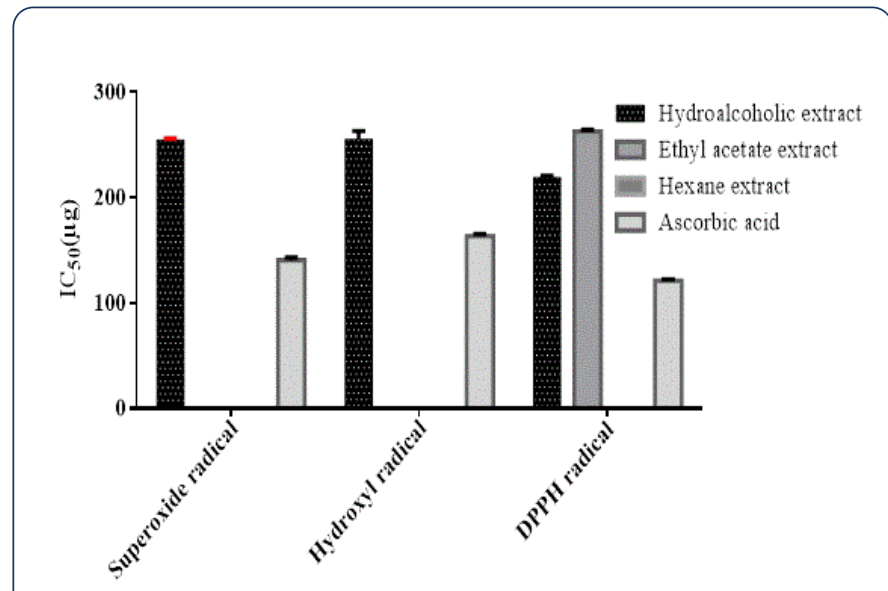

Figure 1 50\% Inhibition concentrations (IC50) of different extracts of $P$. paniculata stem against superoxide, hydroxyl and $\mathrm{DPPH}$ radicals.

\section{Antioxidant activity of Peristrophe paniculata root extracts}

The hydroalcoholic, ethyl acetate and hexane extracts of $P$. paniculata roots were found to possess concentration dependent scavenging activity. The extracts of $P$. paniculata root showed more percentage of inhibition at $320 \mu \mathrm{g}$. The IC50 values for the root extracts were not able to detect because they showed minimal below $50 \%$ inhibition on free radicals production. The percentage of inhibition on superoxide radical of hydroalcoholic, ethyl acetate and hexane extracts of $P$. paniculata root at $320 \mu \mathrm{g}$ were found to be $19.4 \pm 0.25,38.3 \pm$ 0.78 and $25.45 \pm 0.75$ respectively. The percentage of inhibition on hydroxyl radical of hydroalcoholic, ethyl acetate and hexane extracts of $P$. paniculata root at $320 \mu \mathrm{g}$ were found to be $20.8 \pm 0.6,35.1 \pm 0.8$ and $39.3 \pm 0.4$ respectively. The percentage of inhibition on DPPH radical of hydroalcoholic, ethyl acetate and hexane extracts of $P$. paniculata root at 320 $\mu \mathrm{g}$ were found to be $12.28 \pm 0.29,14.35 \pm 0.62$ and $22.1 \pm 0.13$ respectively (Table 6). Among the samples, better DPPH free radical scavenging activity was found in hydroalcoholic root extract of $P$. paniculata.

\section{Discussion}

Free radicals are produced during metabolisms of the body. If they were produced in the normal level, the body produced antioxidants were sufficient to neutralize them without affecting the body [26]. Present day lifestyle are generating more oxidants in the body and the naturally available antioxidants in the body are not sufficient to neutralize excess amount $[26,27]$. The excess production of the oxidants in the body leading to unbalance physiological functions can causing different diseases like lipid peroxidation, DNA damage, atherosclorosis (oxidated LDL is more atherogenic), cancers, neurodegenerative and inflammatory bowel diseases and accelerated aging [28-30]. So, it is important to identify antioxidants without side effects and easily available and consumable [31]. 


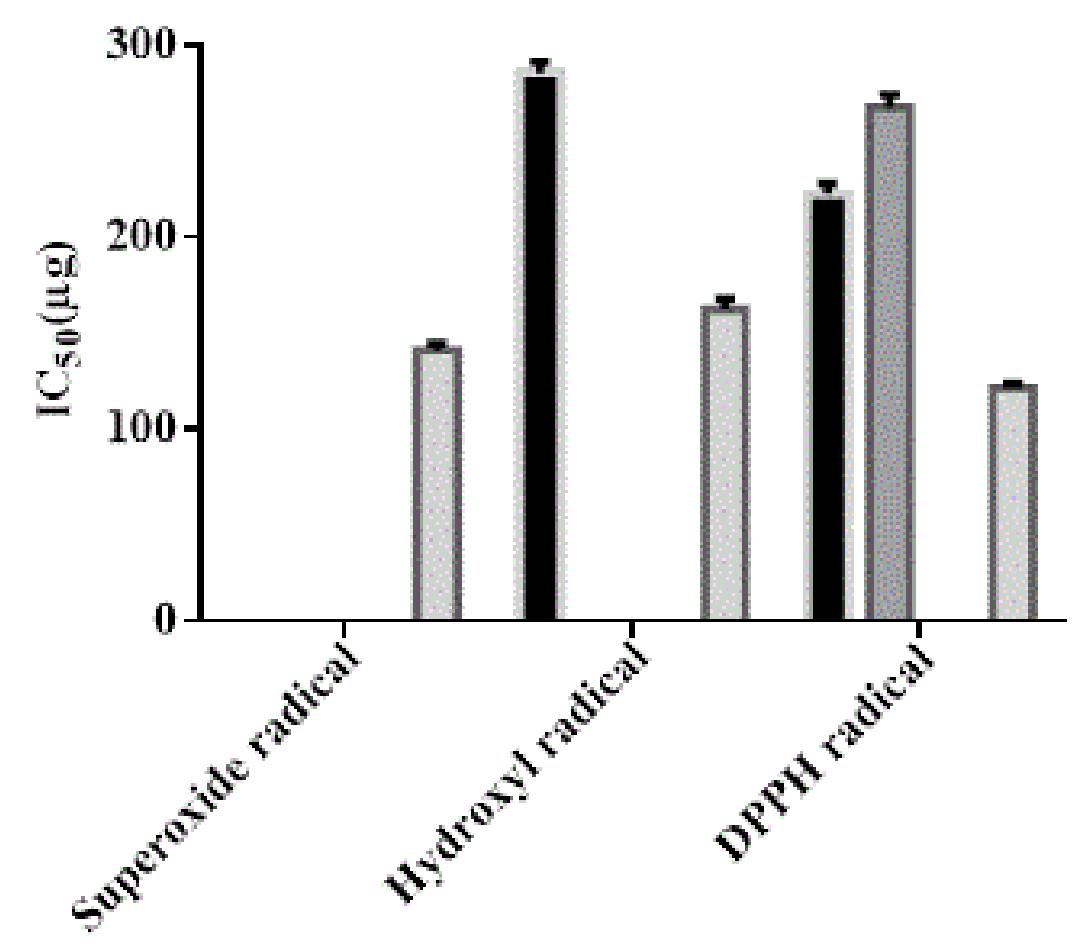

- Hydroalcoholic extract

$\square$ Ethyl acetate extract

- Hexane extract

$\square$ Ascorbic acid

Figure 2 50\% Inhibition concentrations (IC50) of different extracts of P. paniculata leaves against Superoxide, Hydroxyl and DPPH radicals.

Table 5 Concentration dependent percentage inhibition of different extracts of $P$. paniculata leaves on superoxide, hydroxyl and DPPH free radicals.

\begin{tabular}{|c|c|c|c|c|c|c|c|c|c|c|c|c|c|c|c|}
\hline \multirow{2}{*}{$\begin{array}{l}\text { Name of } \\
\text { the } \\
\text { extracts }\end{array}$} & \multicolumn{5}{|c|}{$\%$ of inhibition on superoxide radical } & \multicolumn{5}{|c|}{$\%$ of inhibition on hydroxyl radical } & \multicolumn{5}{|c|}{$\%$ of inhibition on DPPH radical } \\
\hline & $\begin{array}{l}20 \\
\mu g\end{array}$ & $\begin{array}{l}40 \\
\mu \mathrm{g}\end{array}$ & $\begin{array}{l}80 \\
\mu \mathrm{g}\end{array}$ & $\begin{array}{l}160 \\
\mu \mathrm{g}\end{array}$ & $\begin{array}{l}320 \\
\mu \mathrm{g}\end{array}$ & $\begin{array}{l}20 \\
\mu g\end{array}$ & $40 \mu g$ & $80 \mu g$ & $\begin{array}{l}160 \\
\mu \mathrm{g}\end{array}$ & $\begin{array}{l}320 \\
\mu \mathrm{g}\end{array}$ & $20 \mu g$ & $40 \mu g$ & $80 \mu g$ & $\begin{array}{l}160 \\
\mu \mathrm{g}\end{array}$ & $\begin{array}{l}320 \\
\mu \mathrm{g}\end{array}$ \\
\hline $\begin{array}{l}\text { Hexane } \\
\text { extract }\end{array}$ & 0.00 & $\begin{array}{l}3.88 \\
\pm \\
0.2\end{array}$ & $\begin{array}{l}7.5 \\
\pm \\
0.3\end{array}$ & $\begin{array}{l}13.8 \pm \\
0.35\end{array}$ & $\begin{array}{l}23.3 \pm \\
0.42\end{array}$ & $\begin{array}{l}2.32 \\
\pm \\
0.08\end{array}$ & $\begin{array}{l}5.3 \pm \\
0.28\end{array}$ & $\begin{array}{l}11.3 \pm \\
1.23\end{array}$ & $\begin{array}{l}22.3 \pm \\
0.63\end{array}$ & $\begin{array}{l}38.25 \\
\pm 0.72\end{array}$ & $\begin{array}{l}3.4 \pm \\
0.3\end{array}$ & $\begin{array}{l}6.3 \pm \\
1.2\end{array}$ & $\begin{array}{l}12.1 \pm \\
0.83\end{array}$ & $\begin{array}{l}21.82 \\
\pm 0.39\end{array}$ & $\begin{array}{l}39.48 \\
\pm 0.39\end{array}$ \\
\hline $\begin{array}{l}\text { Ethyl } \\
\text { Acetate } \\
\text { extract }\end{array}$ & 0.00 & $\begin{array}{l}2.4 \\
\pm \\
0.3\end{array}$ & $\begin{array}{l}4.9 \\
\pm \\
0.13\end{array}$ & $\begin{array}{l}9.3 \pm \\
0.63\end{array}$ & $\begin{array}{l}17.3 \pm \\
0.8\end{array}$ & 0.0 & $\begin{array}{l}1.45 \\
\pm 0.2\end{array}$ & $\begin{array}{l}3.2 \pm \\
0.15\end{array}$ & $\begin{array}{l}6.8 \pm \\
0.73\end{array}$ & $\begin{array}{l}13.9 \pm \\
0.53\end{array}$ & $\begin{array}{l}2.3 \pm \\
0.5\end{array}$ & $\begin{array}{l}4.8 \pm \\
0.9\end{array}$ & $\begin{array}{l}10.2 \pm \\
0.19\end{array}$ & $\begin{array}{l}21.09 \\
\pm 0.63\end{array}$ & $\begin{array}{l}36.4 \pm \\
0.53\end{array}$ \\
\hline $\begin{array}{l}\text { Hydro- } \\
\text { alcoholic } \\
\text { extract }\end{array}$ & $\begin{array}{l}2.2 \\
\pm \\
0.3\end{array}$ & $\begin{array}{l}6.8 \\
\pm \\
0.1\end{array}$ & $\begin{array}{l}12.4 \\
\pm \\
0.8\end{array}$ & $\begin{array}{l}23.5 \pm \\
0.2\end{array}$ & $\begin{array}{l}38.4 \pm \\
0.68\end{array}$ & $\begin{array}{l}4.62 \\
\pm \\
1.08\end{array}$ & $\begin{array}{l}9.2 \pm \\
0.63\end{array}$ & $\begin{array}{l}17.82 \\
\pm 0.83\end{array}$ & $\begin{array}{l}34.6 \pm \\
0.3\end{array}$ & $\begin{array}{l}53.2 \pm \\
0.61\end{array}$ & $\begin{array}{l}5.23 \pm \\
0.98\end{array}$ & $\begin{array}{l}11.3 \pm \\
0.19\end{array}$ & $\begin{array}{l}21.23 \\
\pm 0.25\end{array}$ & $\begin{array}{l}39.9 \pm \\
0.93\end{array}$ & $\begin{array}{l}58.2 \pm \\
0.69\end{array}$ \\
\hline $\begin{array}{l}\text { Ascorbic } \\
\text { acid }\end{array}$ & $\begin{array}{l}5.82 \\
\pm \\
0.39\end{array}$ & $\begin{array}{l}11.6 \\
\pm \\
0.89\end{array}$ & $\begin{array}{l}25.7 \\
\pm \\
1.2\end{array}$ & $\begin{array}{l}58.64 \\
\pm 0.18\end{array}$ & $\begin{array}{l}75.36 \\
\pm 0.76\end{array}$ & $\begin{array}{l}6.39 \\
\pm \\
0.84\end{array}$ & $\begin{array}{l}11.93 \\
\pm \\
0.45\end{array}$ & $\begin{array}{l}23.6 \pm \\
0.71\end{array}$ & $\begin{array}{l}47.89 \\
\pm 0.53\end{array}$ & $\begin{array}{l}73.34 \\
\pm 0.62\end{array}$ & $\begin{array}{l}10.26 \\
\pm 0.28\end{array}$ & $\begin{array}{l}20.47 \\
\pm 0.75\end{array}$ & $\begin{array}{l}36.9 \pm \\
0.55\end{array}$ & $\begin{array}{l}62.31 \\
\pm 1.26\end{array}$ & $\begin{array}{l}78.08 \\
\pm 0.40\end{array}$ \\
\hline
\end{tabular}

Table 6 Concentration dependent percentage inhibition of different extracts of $P$. paniculata roots on superoxide, hydroxyl and DPPH free radicals.

\begin{tabular}{|c|c|c|c|c|c|c|c|c|c|c|c|c|c|c|c|}
\hline \multirow{2}{*}{$\begin{array}{l}\text { Name of } \\
\text { the } \\
\text { extracts }\end{array}$} & \multicolumn{5}{|c|}{$\%$ of inhibition on superoxide radical } & \multicolumn{5}{|c|}{$\%$ of inhibition on hydroxyl radical } & \multicolumn{5}{|c|}{$\%$ of inhibition on DPPH radical } \\
\hline & $\begin{array}{l}20 \\
\mu \mathrm{g}\end{array}$ & $\begin{array}{l}40 \\
\mu \mathrm{g}\end{array}$ & $\begin{array}{l}80 \\
\mu \mathrm{g}\end{array}$ & $\begin{array}{l}160 \\
\mu \mathrm{g}\end{array}$ & $\begin{array}{l}320 \\
\mu \mathrm{g}\end{array}$ & $\begin{array}{l}20 \\
\mu \mathrm{g}\end{array}$ & $40 \mu \mathrm{g}$ & $\begin{array}{l}80 \\
\mu \mathrm{g}\end{array}$ & $\begin{array}{l}160 \\
\mu \mathrm{g}\end{array}$ & $\begin{array}{l}320 \\
\mu \mathrm{g}\end{array}$ & $20 \mu \mathrm{g}$ & $40 \mu g$ & $\begin{array}{l}80 \\
\mu \mathrm{g}\end{array}$ & $\begin{array}{l}160 \\
\mu \mathrm{g}\end{array}$ & $\begin{array}{l}320 \\
\mu \mathrm{g}\end{array}$ \\
\hline
\end{tabular}




\begin{tabular}{|c|c|c|c|c|c|c|c|c|c|c|c|c|c|c|c|}
\hline $\begin{array}{l}\text { Hexane } \\
\text { extract }\end{array}$ & 0.00 & $\begin{array}{l}1.85 \\
\pm \\
0.6\end{array}$ & $\begin{array}{l}4.3 \\
\pm \\
0.2\end{array}$ & $\begin{array}{l}8.9 \\
\pm 0.53\end{array}$ & $\begin{array}{l}19.4 \\
\pm 0.25\end{array}$ & 0.00 & $\begin{array}{l}2.23 \\
\pm 0.53\end{array}$ & $\begin{array}{l}5.1 \\
\pm \\
0.29\end{array}$ & $\begin{array}{l}11.23 \\
\pm 1.89\end{array}$ & $\begin{array}{l}20.8 \\
\pm 0.6\end{array}$ & 0.00 & 0.00 & $\begin{array}{l}1.83 \\
\pm \\
0.28\end{array}$ & $\begin{array}{l}5.23 \\
\pm 0.5\end{array}$ & $\begin{array}{l}12.28 \\
\pm 0.24\end{array}$ \\
\hline $\begin{array}{l}\text { Ethyl } \\
\text { Acetate } \\
\text { extract }\end{array}$ & 0.00 & $\begin{array}{l}5.2 \\
\pm \\
0.06\end{array}$ & $\begin{array}{l}11.2 \\
\pm \\
0.3\end{array}$ & $\begin{array}{l}20.8 \\
\pm 0.69\end{array}$ & $\begin{array}{l}38.3 \\
\pm 0.78\end{array}$ & 0.00 & $\begin{array}{l}3.46 \\
\pm 0.52\end{array}$ & $\begin{array}{l}7.8 \\
\pm \\
0.91\end{array}$ & $\begin{array}{l}18.3 \\
\pm 0.35\end{array}$ & $\begin{array}{l}35.1 \\
\pm 0.8\end{array}$ & 0.00 & 0.00 & $\begin{array}{l}2.5 \\
\pm \\
0.63\end{array}$ & $\begin{array}{l}6.8 \\
\pm 0.39\end{array}$ & $\begin{array}{l}14.35 \\
\pm 0.62\end{array}$ \\
\hline $\begin{array}{l}\text { Hydro- } \\
\text { alcoholic } \\
\text { extract }\end{array}$ & 0.00 & $\begin{array}{l}3.2 \\
\pm \\
0.52\end{array}$ & $\begin{array}{l}5.8 \\
\pm \\
0.25\end{array}$ & $\begin{array}{l}13.2 \\
\pm 0.35\end{array}$ & $\begin{array}{l}25.4 \\
\pm 0.95\end{array}$ & $\begin{array}{l}2.2 \\
\pm \\
0.78\end{array}$ & $\begin{array}{l}5.3 \\
\pm 0.28\end{array}$ & $\begin{array}{l}12.6 \\
\pm \\
0.73\end{array}$ & $\begin{array}{l}25.2 \\
\pm 0.13\end{array}$ & $\begin{array}{l}39.3 \\
\pm 0.4\end{array}$ & 0.00 & $\begin{array}{l}2.63 \\
\pm 0.05\end{array}$ & $\begin{array}{l}5.8 \\
\pm \\
0.32\end{array}$ & $\begin{array}{l}10.92 \\
\pm 0.43\end{array}$ & $\begin{array}{l}25.19 \\
\pm 0.13\end{array}$ \\
\hline $\begin{array}{l}\text { Ascorbic } \\
\text { acid }\end{array}$ & $\begin{array}{l}5.82 \\
\pm \\
0.39\end{array}$ & $\begin{array}{l}11.6 \\
\pm \\
0.89\end{array}$ & $\begin{array}{l}25.7 \\
\pm \\
1.2\end{array}$ & $\begin{array}{l}58.64 \\
\pm 0.18\end{array}$ & $\begin{array}{l}75.36 \\
\pm 0.76\end{array}$ & $\begin{array}{l}6.39 \\
\pm \\
0.84\end{array}$ & $\begin{array}{l}11.93 \\
\pm 0.45\end{array}$ & $\begin{array}{l}23.6 \\
\pm \\
0.71\end{array}$ & $\begin{array}{l}47.89 \\
\pm 0.53\end{array}$ & $\begin{array}{l}73.34 \\
\pm 0.62\end{array}$ & $\begin{array}{l}10.26 \\
\pm 0.28\end{array}$ & $\begin{array}{l}20.47 \\
\pm 0.75\end{array}$ & $\begin{array}{l}36.9 \\
\pm \\
0.55\end{array}$ & $\begin{array}{l}62.31 \\
\pm 1.26\end{array}$ & $\begin{array}{l}78.08 \\
\pm 0.40\end{array}$ \\
\hline
\end{tabular}

Medicinal plants have attracted great attention for their potent biological activities, no side effects and economic viability over the past years. The biological activities (properties) of medicinal plants may be due to the presence of diverse group of chemical compounds like steroids, glycosides, phenolics, glycosides, anthocyanins, flavonoids etc. [32-34]. The search of biologically active compounds from medicinal plants has always been of great interest to scientists looking for new sources of useful drugs against different hazardous diseases. Many plant species have been investigated in the search for antioxidants but generally there is still a demand to find more information concerning the antioxidant potential of plant species $[18,21,35,36]$. Many studies have shown that natural antioxidants in medicinal plants closely related to their biofunctionalities, such as the prevention or suppression of aging and many diseases associated with oxidative stress, cancer, cardiovascular diseases, rheumatoid arthritis, autoimmune diseases and AIDS [37].

The present work carried out to identify variability in the presence of phytochemical compounds in different parts and antioxidant capacity of different parts of traditional medicinal plant, P. paniculata and we succeeded in identification of the medicinal value of $P$. paniculata scientifically. The phytochemical analysis of different parts extracts showed presence of different phytochemical compounds in them (Tables 1-3). The hexane extracts of $P$. paniculata showed mainly presences of steroids, terpenoids, oils and carbohydrates. Ethyl acetate extracts showed mainly presences of glycosides, flavanoids, alkaloids, phenols, tannins etc. Hydralcoholic extracts showed the presences of maximum compounds in them but have little variability compared to hexane and ethyl acetate extracts. The variation in the presence of phytochemical compounds may be due to their solubility in the solvents used for their extraction [38]. The presence of compounds may also varies from one region to other regions, because of their geographical variation.

The concentration dependent percentage inhibition of tested plants extracts varies from one free radical to other on tested free radicals. The hydralcoholic extract showed more activity on superoxide free radical and DPPH free radicals, hexane extract showed less activity. Among three parts extracts of $P$. paniculata, stem extracts showed more percentage inhibition on free radicals and the phytochemical analysis of different parts extracts showed presence of different phytochemical compounds in them. In recent decades, there were many scientific reports on medicinal plants about their antioxidant activities $[25,38]$. So, the results of the present study provide scientific evidence for its traditional medicinal uses.

\section{Acknowledgements}

The authors thanking to authorities of MAM College of Pharmacy for providing the necessary laboratory chemicals and equipments.

\section{Conflict of Interest}

The authors have none to declare.

\section{References}

1. Sarker SD, Nahar L (2007) Chemistry for pharmacy students general, organic and natural product chemistry. England: John Wiley and Sons, p: 369.

2. Doughari JH, Human IS, Bennade S, Ndakidemi PA (2009) Phytochemicals as chemotherapeutic agents and antioxidants: possible solution to the control of antibiotic resistant verocytotoxin producing bacteria. J Med Plants Res 3: 839-848.

3. Liu RH (2004) Potential synergy of phytochemicals in cancer prevention: mechanism of Action. J Nutr 134: 3479S-3485S.

4. Saganuwan AS, Onyeyili PA (2010) Biochemical effects of aqueous leaf extract of abrus precatorius (jecquirity bean) in swiss albino mice. Herba Pol 56: 63-80.

5. Galm, U, Shen B (2007). Natural product drug discovery: the times have never been better. ACS Chem Biol 14: 1098-1104.

6. Firn R (2010) Nature's Chemicals. Oxford University Press, Oxford.

7. Kris Etherton PM, Hecker KD, Bonanome A, Coval SM, Binkoski $A E$, et al. (2002) Bioactive compounds in foods: their role in the prevention of cardiovascular disease and cancer. Am J Med 113:71S-88S.

8. Khare CP (2007) Indian medicinal plants an illustrated dictionary. Springer.

9. Kirtikar KR, Basu BD (1918) Indian medicinal plants. Allahabad: Leader Press. 
10. Pullaiah T, Ramakrishnaiah V, Sandhya Rani S, Rao PN (2000) Flora of guntur district, andhra pradesh, india. Regency Publications, New Delhi, p: 271.

11. Pullaiah T, Prabhakar C, Ravi Prasad Rao B (1998) Flora of medak district, andhra pradesh, india. Daya Publishing House, New Delhi.

12. Pullaiah T (2006) Encyclopaedia of world medicinal plants. Regency Publications, New Delhi 1: 1492-1493.

13. Abdulazeez AM (2012) Antihypertensive and anticancer activities of extracts of Peristrophe bicalyculata (Retz) Nees and their effects on expressions of angiotensin-converting enzyme and endothelial nitric oxide synthase. Ph.D Thesis. Ahmadu Bello University, Biochemistry Department.

14. Siddiqui ZA, Mahamood I (1994) Culture of Paecilomyces lilacinus on Leaf Extracts and Leaf Residues for Nematode Control. Bioresour Technol 49: 187.

15. Rathi A, Rao C, Khatoon S, Mehrotra S (2003) Ethnopharmacological evaluation of Peristrophe bicalyculata Nees for anti-inflammatory and analgesic activity. Nat Prod Sci 9: 195-199.

16. Ogunwande IA, Walker TM, Bansal A, Setzer WN, Essien EE (2010) Essential oil constituents and biological activities of Peristrophe bicalyculata and Borreria verticillata. Nat Prod Comm 5: 1815-1818.

17. Janakiraman N, Sahaya SS, Johnson M (2012) Anti-bacterial studies on Peristrophe bicalyculata (Retz.) Nees. Asian Pac J Trop Biomed 1: 2.

18. Ganga Rao B, Venkateswara Rao Y, Mallikarjuna Rao T (2013) Hepatoprotective and antioxidant capacity of Melochia corchorifolia extracts. Asian Pac J Trop Med 6: 537-543.

19. Ayoola GA, Coker HAB, Adesegun SA, Adepoju-Bello AA, Obaweya K, et al. (2008) Phytochemical screening and antioxidant activities of some selected medicinal plants used for malaria therapy in Southwestern Nigeria. Trop J Pharm Res 7: 1019-1024.

20. Prashant T, Bimlesh K, Mandeep K, Gurpreet K, Harleen K (2011) Phytochemical screening and extraction: A review. Int Pharm Sci 1: 98-106.

21. Mallikarjuna RT, Ganga RB, Venkateswara RY (2012) Antioxidant activity of Spilanthes acmella extracts. International Journal of Phytopharmacology 3: 216-220.

22. Mc Cord JM, Fridovich I (1969) Superoxide dismutase: an enzymatiic function for erythrocuprein (hemocuprien). J Biol Chem 244: 6049-6055.

23. Elizabeth K, Rao MNA (1990) Oxygen radical scavenging activity of curcumin. Int J Pharm 58: 237-240.

24. Braca A, Fico G, Morelli I, De Simone F, Tome F, et al. (2003) Antioxidant and free radical scavenging activity of flavonol glycosides from different Aconitum species. J Ethnopharmacol 86: 63-67.

25. Anita M, Purnima A, Madhavan V (2011) In vitro antioxidant activity and HPTLC Studies on the roots and rhizomes of Smilax zeylanica I. (smilacaceae). Int J Pharm Pharm Sci 3: 192-195.

26. Finkel T, Holbrook NJ (2000) Oxidants, oxidative stress and the biology of aging. Nature 408: 239-247.

27. Valko M, Leibfritz D, Moncol J, Cronin MT, Mazu M, et al. (2007) Free radicals and antioxidants in normal physiological functions and human disease. Int J Biochem Cell Biol 39: 44-84.

28. Halliwell B, Gutteridge JMC, Cross CE (1992) Free radicals, antioxidants, and human disease: where are we now?. J Lab Clin Med 119: 598-619.

29. Pietta PG (2000) Flavanoids as antioxidants. J Nat Prod 63: 1035-1042.

30. Wang JH, Wang $\mathrm{HZ}$, Zhang $\mathrm{M}$, Zhang SH (2002) Effect of antiaging Lycium barbarum polysaccharide. Acta Pharmacol Sin 24: 189-191.

31. Lobo V, Patil A, Phatak A, Chandra N (2010) Free radicals, antioxidants and functional foods: Impact on human health. Pharmacog Rev 4: 118-126.

32. Exarchou V, Nenadis N, Tsimidou M, Gerothanassis IP, Troganis A, et al. (2002) Antioxidant activities and phenolic composition of extracts from Greek oregano, Greek sage and summer savory. J Agric Food Chem 50: 5294-5299.

33. Alzoreky NS, Nakahara K (2003) Antimicrobial activity of extracts from some edible plants commonly consumed in Asia. Int J Food Microbiol 80: 223-230.

34. Amensour M, Sendra E, Jamal A, Bouhdid S, Pérez-Alvarez JA, et al. (2009) Total phenolic content and antioxidant activity of myrtle (Myrtus communis) extracts. Nat Prod Commun 4: 819-824.

35. Jayashree G, Kurup MG, Sudarslal S, Jacob VB (2003) Antioxidant activity of Centella asiatica on lymphoma-bearing mice. Fitoterapia 74: 431-434.

36. Katerere DR, Eloff JN (2008) Anti-bacterial and anti-oxidant activity of Hypoxis hemerocallidea (Hypoxidaceae): can leaves be substituted for corms as a conservation strategy?. S Afr J Bot 74: 613-616.

37. Ames BN, Shigenaga MK, Hagen TM (1993) Oxidants, antioxidants, and the degenerative diseases of aging. PNAS 90: 7915-7922.

38. Abah SE, Egwari LO (2011) Methods of Extraction and Antimicrobial Susceptibility Testing of Plant Extracts. African J Basic \& Appl Sci 3: 205-209. 